\title{
Smoking at time of CIS increases the risk of clinically definite multiple sclerosis
}

\author{
Roos M. van der Vuurst de Vries ${ }^{1}$. Julia Y. Mescheriakova ${ }^{1} \cdot$ Tessel F. Runia $^{1} \cdot$ Theodora A. M. Siepman $^{1}$. \\ Beatrijs H. A. Wokke ${ }^{1}$. Johnny P. A. Samijn ${ }^{2}$ Rogier Q. Hintzen ${ }^{1}$
}

Received: 15 December 2017 / Revised: 29 January 2018 / Accepted: 30 January 2018 / Published online: 20 February 2018

(c) The Author(s) 2018. This article is an open access publication

\begin{abstract}
Background Cigarette smoking is a modifiable risk factor that influences the disease course of patients with multiple sclerosis (MS). However, in patients with a clinically isolated syndrome (CIS), there are conflicting results about the association between smoking and the risk of a subsequent MS diagnosis. The aim of this study was to determine the risk of clinically definite MS (CDMS) in smoking and non-smoking patients at time of a first demyelinating event.

Methods Two hundred and fifty patients, aged 18-50 years, were included in our prospective CIS cohort. At time of the first neurological symptoms, patients completed a questionnaire about smoking habits. Cox regression analyses were performed to calculate univariate and multivariate hazard ratios for CDMS diagnosis in smoking and non-smoking CIS patients.

Results One hundred and fourteen (46\%) CIS patients were diagnosed with CDMS during a mean follow-up of 58 months. In total, 79 (32\%) patients smoked at time of CIS. Sixty-seven \% of the smoking CIS patients were diagnosed with CDMS during follow-up compared to $36 \%$ of the non-smoking CIS patients $(p<0.001)$. Smoking at time of CIS was an independent predictor for CDMS diagnosis (HR 2.3; $p=0.002$ ). Non-smoking CIS patients who had a history of smoking did not have a higher risk for CDMS than those who had never smoked.

Conclusions Smoking at time of CIS was an independent risk factor for a future CDMS diagnosis. This is an additional argument to quit smoking at time of the first attack of suspected MS.
\end{abstract}

Keywords Clinically isolated syndrome $\cdot$ Multiple sclerosis $\cdot$ Smoking

\section{Introduction}

Multiple sclerosis (MS) is a chronic inflammatory autoimmune disease, influenced by environmental factors in genetically susceptible individuals [1]. This results in demyelination, axonal loss, and neurodegeneration [1-3]. The course of MS is heterogeneous in severity and prognosis [1].

One of the environmental factors influencing the course of MS is cigarette smoking [4]. Studies in MS patients and healthy controls consistently provide evidence that both active and passive smoking result in an increased risk of MS and disease progression [4-7]. Smoking does not only

Rogier Q. Hintzen

r.hintzen@erasmusmc.nl

1 Department of Neurology, MS Centre ErasMS, Erasmus MC, P.O. Box 2040, 3000 CA Rotterdam, The Netherlands

2 Department of Neurology, Maasstad Hospital, Rotterdam, The Netherlands increase MS risk, it also shortens the time to the secondary progressive phase of MS (SPMS) [8, 9]. It has been shown that after cessation of smoking, negative effects slowly decrease, independent of the cumulative dose of smoking $[8,10]$.

Studies show that the risk of MS associated with HLA genotypes is influenced by smoking. This interaction leads to a much stronger effect on MS risk than the cumulative effect of genetic risk factors and smoking together [11, 12].

In the majority of cases (85-90\%), MS starts as a clinically isolated syndrome (CIS), followed by novel episodes of neurological symptoms resulting from inflammation of the central nervous system (CNS). However, a CIS attack can also remain a single event [13].

Only a few studies are available in CIS patients that investigated the effect of smoking on subsequent MS risk. Firm conclusions in these studies are hampered by several methodologic issues such as low patient numbers, retrospective designs [14-16], or considerable numbers of CIS patients 
treated with interferon beta $[17,18]$. On the other hand, several studies in MS patients do suggest a link between smoking and MS progression [7]. Therefore, we determined the effect of smoking on clinically definite MS (CDMS) risk in a large prospective cohort of predominantly untreated CIS patients. It is important to know if smoking is associated with a future MS diagnosis, as smoking is up to now, one of the few modifiable risk factors for disease progression in MS.

\section{Methods}

\section{Patients}

Data were collected prospectively from patients with CIS at the Neurology Department of Erasmus MC University Hospital in Rotterdam, a tertiary referral centre for patients with MS. Data collection was in collaboration with several regional hospitals in The Netherlands. Included patients had their first symptoms between May 2006 and June 2017. Patients were aged between 18 and 50 years, with no history of previous neurological symptoms suggestive of CNS demyelination. CIS patients were included within 6 months following the first neurological symptoms. Patients with alternative diagnoses were excluded from the analyses. At baseline, a magnetic resonance imaging (MRI) scan and routine laboratory tests were utilized to rule out alternative diagnoses [19]. Following inclusion, patients were reassessed at least annually at the Neurological Outpatient Department.

\section{Questionnaire}

At baseline, CIS patients completed a questionnaire to gather information about smoking habits, including when they first started smoking, non- or reduced-smoking periods and how many cigarettes were smoked within these periods. Using results of the questionnaire, we were able to calculate the pack-years per patient.

\section{Standard protocol approvals, and patient consent}

This study was approved by the Medical Ethics Committee of Erasmus MC Rotterdam. Written informed consent was obtained from all patients.

\section{Definitions}

A relapse was defined as new symptoms or subacute worsening of existing symptoms after 30 days of improvement, or stable disease and no evidence of alternative diagnosis. Symptoms had to exist longer than $24 \mathrm{~h}$ and not to be preceded by fever [20]. All exacerbations were confirmed by neurological examination. CDMS was defined as clinical dissemination in space and time with two exacerbations and (para) clinical evidence of two separate lesions, as described by Poser et al. [21]. Patients who were diagnosed with CDMS during follow-up are referred to as CIS-CDMS and patients who remained CIS are referred to as CIS-CIS. Expanded Disability Status Scale (EDSS) scores were performed annually when patients were diagnosed with CDMS [22]. EDSS performed within 3 months after a relapse were not used in the analyses. Follow-up was calculated by subtracting CIS date from the last visit date. Patients were defined as smokers when they were smoking regularly at time of CIS. Non-smokers were those who did not smoke at time of CIS. Patients were defined as ex-smokers when they were not smoking at time of CIS, but did have a history of smoking in the years prior to CIS. To calculate pack-years, the number of years smoked was multiplied by the number of cigarettes smoked per day/20 in that period.

\section{Statistical analysis}

Statistical analyses were done using SPSS, version 21.0 (SPSS Inc) for Windows and GraphPad Prism5 (GraphPad) for Windows. Nominal data comparison between groups was done using Chi-square or Fisher's exact test [gender, type of clinical onset, oligoclonal bands (OCB), $\geq 9 \mathrm{~T} 2$ lesions on baseline MRI, disease modifying therapy (DMT) at time of CIS, smoking at time of CIS, SPMS, and alcohol use]. The Kolmogorov-Smirnov test was performed to assess normality of data distribution. To compare continuous data, we applied a two-tailed $t$ test (age at onset and follow-up time) or when the data were non-parametric, a Mann-Whitney $U$ test (time from CIS to CDMS and pack-years). Time to second attack was calculated from onset of the first symptoms. Cox-proportional hazard regression analyses were used to calculate univariate and multivariate hazard ratios (HR). Patients who did not have a second attack during followup were considered as censored observations. Hazard ratios were also obtained for time to EDSS 4.0 and time to EDSS 6.0. $p$ values less than 0.05 were considered significant.

\section{Results}

\section{Patient characteristics}

We included 250 patients who completed the baseline questionnaire about smoking at time of CIS. Out of these 250 CIS patients, 114 (46\%) patients had a second relapse and were diagnosed with CDMS during a mean follow-up time of 58.1 months (SD 35.9). 
The median time (interquartile range; IQR) from CIS to CDMS was 23.3 months (8.9-44.3). The median time (IQR) between the first neurological symptoms and inclusion in the study was 1.2 months (0.3-2.9 months).

Fifty-seven (23\%) patients who were not yet diagnosed with CDMS were treated with DMT. The patient characteristics are shown in Table 1.

\section{Smokers vs non-smokers}

In total, 79 out of $250(32 \%)$ patients smoked at time of CIS. Fifty-three out of 79 (67\%) smoking CIS patients were diagnosed with CDMS during follow-up compared to 61 out of $171(36 \%)$ in the non-smoking CIS patients $(p<0.001)$. The number of pack-years was higher in the group that was diagnosed with CDMS (CIS-CDMS) during follow-up than in the group that remained CIS (CIS-CIS) (median (IQR) CIS-CDMS vs CIS-CIS: $2.4(0.0-11.9)$ vs $0.0(0.0-2.7)$ $p=0.004)$ (Fig. 1).

There were no differences between smokers and nonsmokers in gender, localisation of first symptoms, age, OCB in CSF or MRI characteristics at baseline. Table 2 shows patient characteristics for smokers and non-smokers.

\section{Association of smoking at time of CIS with a shorter time to CDMS}

Patients who smoked at time of CIS had a shorter time to CDMS diagnosis than patients who were not active smokers (univariate hazard ratio; HR $2.1 p<0.001$ ) (Fig. 2). Corrections were applied for multiple variables that are associated with a second attack (OCB in CSF, more than nine T2 lesions, gadolinium enhancing lesions on baseline MRI, and

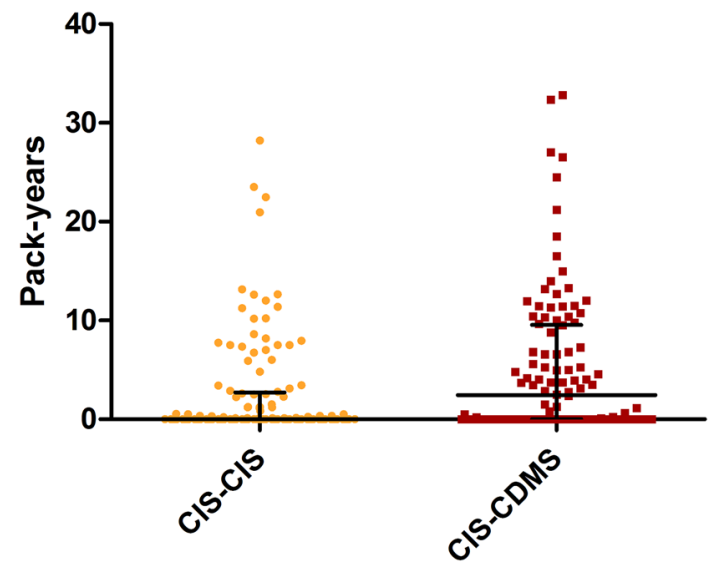

Fig. 1 Pack-years in CIS patients. Comparison of pack-years between CIS-CIS and CIS-CDMS patients. Horizontal lines and error bars indicate median and IQR

optic neuritis as first symptom, no DMT before CDMS). After these adjustments, multivariate COX regression analysis showed smoking as an independent predictor for a second attack. The HR was $2.3(p=0.002)$.

In a sub-analysis, we excluded the 50 CIS patients who had less than 2 years of follow-up. After exclusion, the results remained the same, HR $2.0(p<0.001)$.

Fifty-seven (23\%) of the patients received DMT before CDMS diagnosis. When we excluded these patients, the HR was unchanged $2.0(p=0.001)$.

\section{Smoking in the past}

In the group of patients that did not smoke at time of CIS $(n=171), 63(37 \%)$ patients had a history of smoking in

Table 1 Patient characteristics (CIS-CDMS vs CIS-CIS patients)

\begin{tabular}{|c|c|c|c|c|}
\hline & CIS patients $(n=250)$ & CIS-CDMS $(n=114)$ & CIS-CIS $(n=136)$ & $p$ value $^{\mathrm{a}}$ \\
\hline Female sex, no. (\%) & 189 (75.6) & $92(80.7)$ & $97(71.3)$ & 0.09 \\
\hline Age, mean (SD), years & $33.6(8.3)$ & $32.6(7.9)$ & $34.5(8.5)$ & 0.07 \\
\hline Follow-up time, mean (SD), months & $58.1(35.9)$ & $72.3(30.4)$ & $46.3(36.0)$ & $<0.01$ \\
\hline \multicolumn{5}{|l|}{ Type of clinical onset, no. (\%) } \\
\hline Optic nerve & $88(35.2)$ & $33(28.9)$ & $55(40.4)$ & 0.06 \\
\hline Spinal cord & $90(36.0)$ & $45(39.5)$ & $45(33.1)$ & 0.30 \\
\hline Other localization & $72(28.8)$ & $36(31.6)$ & $36(26.5)$ & 0.37 \\
\hline $\mathrm{OCB},(>1$ band $),(\%)$ & $113(73.9)$ & $63(81.8)$ & $50(65.8)$ & 0.02 \\
\hline$\geq 9$ lesions on $\mathrm{T} 2$-weighted images, no. (\%) & $96(38.6)$ & $53(46.9)$ & $43(31.6)$ & 0.01 \\
\hline DMT at time of CIS, no. (\%) & $57(22.8)$ & $26(22.8)$ & $31(22.8)$ & 1.00 \\
\hline Smoking at time of CIS, no. (\%) & $79(31.6)$ & $53(46.5)$ & $26(19.1)$ & $<0.01$ \\
\hline Pack-years at time of CIS, median (IQR) & $1.0(0.0-5.9)$ & $2.4(0.0-9.5)$ & $0.0(0.0-2.7)$ & $<0.01$ \\
\hline
\end{tabular}

CIS clinically isolated syndrome, $C I S-C D M S$ patients who are diagnosed with CDMS during follow-up after CIS defined by Poser criteria, $C I S-$ $C I S$ not diagnosed with CDMS, na not applicable, $O C B$ oligoclonal bands

${ }^{\text {a }} P$ value calculated between CIS-CDMS and CIS-CIS 
Table 2 Patient characteristics (smoking vs non-smoking CIS patients)

\begin{tabular}{lccr}
\hline & $\begin{array}{l}\text { Smoking CIS } \\
\text { patients }(n=79)\end{array}$ & $\begin{array}{c}\text { Non-smoking CIS } \\
\text { patients }(n=171)\end{array}$ & $p$ value \\
\hline Female sex, no. (\%) & $58(73.4)$ & $131(76.6)$ & 0.59 \\
Age, mean (SD), years & $33.9(7.7)$ & $33.5(8.5)$ & 0.67 \\
Follow-up time, mean (SD), months & $60.7(30.7)$ & $57.0(38.1)$ & 0.45 \\
Type of clinical onset, no. (\%) & & \\
Optic nerve & $25(31.6)$ & $63(36.8)$ & 0.42 \\
Spinal cord & $29(36.7)$ & $61(35.7)$ & 0.87 \\
Other localization & $25(31.6)$ & $47(26.3)$ & 0.50 \\
OCB, (> 1 band), (\%) & $43(75.4)$ & $70(72.9)$ & 0.73 \\
$\geq 9$ lesions on T2-weighted images, no. (\%) & $33(42.3)$ & $63(36.8)$ & 0.41 \\
DMT at time of CIS, no. (\%) & $24(30.4)$ & $33(19.3)$ & $\mathbf{0 . 0 5}$ \\
CDMS, no. (\%) & $53(67.1)$ & $61(35.7)$ & $<\mathbf{0 . 0 1}$ \\
SPMS, no. (\%) & $3(3.8)$ & $5(2.9)$ & 0.72 \\
Alcohol use, no. of patients $(\%)$ & $55(69.6)$ & $79(46.2)$ & $<\mathbf{0 . 0 1}$ \\
\hline
\end{tabular}

$C I S$ clinically isolated syndrome, $O C B$ oligoclonal bands

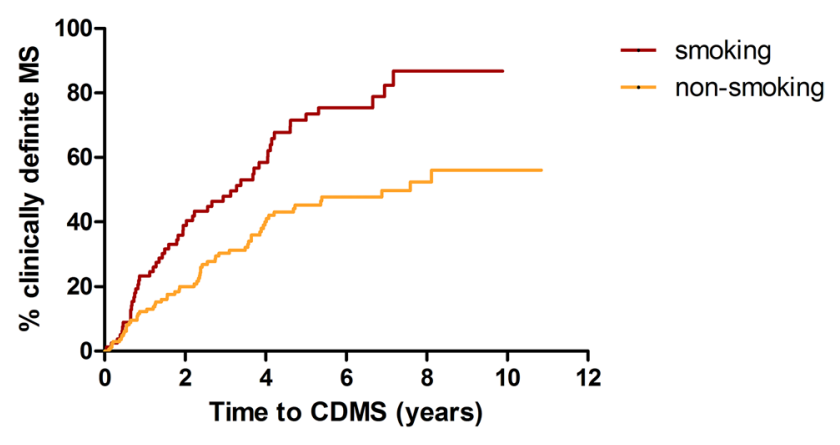

Fig. 2 Time from CIS to CDMS in smoking and non-smoking patients. Kaplan-Meier curve for time from CIS to CDMS for smoking and non-smoking patients at time of CIS [log-rank test $p<0.001)]$

the past (ex-smokers). Smoking in the past did not predict CDMS diagnosis in the group of non-smoking CIS patients (HR 0.64, $p=0.12$ ). Furthermore, in this nonsmoking group $(n=171)$, the number of pack-years was not correlated with time to CDMS [HR per pack-years: 0.96 $(p=0.31)]$.

\section{Smoking at time of CIS and disability later in the disease}

In this cohort, we collected EDSS data from 96/114 (84\%) patients who were diagnosed with CDMS. Nineteen patients reached an EDSS of 4.0 and eight patients an EDSS of 6.0 during follow-up. Six out of these eight patients who reached an EDSS score of 6.0 or more were smoking at time of CIS. The HRs for both EDSS scores were not significant [HR for EDSS 4.0: $1.9(p=0.18)$ and HR for EDSS 6.0: $4.1(p=0.09)]$. However, there was a trend towards faster disability progression in CDMS patients who were smoking at time of CIS.

\section{Discussion}

In this prospective study of patients included after a first attack of suspected MS, we determined the risk of CDMS in a cohort of 250, mostly untreated smoking and non-smoking CIS patients. We demonstrated that smoking at time of CIS is associated with a shorter time to a second clinical attack and, therefore, an earlier diagnosis of CDMS.

Smoking is a well-established risk factor for MS and disease progression after MS diagnosis [4, 5]. This association is also found in other auto-inflammatory diseases such as rheumatoid arthritis (RA) and systemic lupus erythematosus (SLE) [23, 24].

Studies investigating the influence of smoking on MS risk in first attack patients have remained inconclusive, as described recently in a systematic review [25]. Three studies were relatively small and used retrospective data [14-16]. Other studies were large, but patients were treated with interferon beta immediately after CIS or 2 years after CIS, $[17,18]$. Interferon beta treatment could have postponed MS diagnosis [26]. Therefore, the potential correlation between smoking and MS risk may be overshadowed by this diseasepostponing therapy.

Compared to these studies, the present study has less confounding factors, it has a prospective design and only a small proportion of patients was treated with DMT before CDMS diagnosis.

In the multivariate analysis, we corrected our results for currently known predictors for CDMS diagnosis (large number of $\mathrm{T} 2$ lesions, contrast enhancing lesions on baseline 
MRI, unique OCB in CSF and localization of CIS) and for DMT before CDMS diagnosis [27]. After these corrections, smoking remained clearly predictive for CDMS diagnosis. Therefore, smoking status can potentially improve prediction of a future CDMS diagnosis in CIS patients. Accurately predicting CDMS diagnosis is important to prevent unnecessary treatment of patients with low disease activity [28].

The fact that smoking in the past in current non-smoking CIS patients was not associated with CDMS suggests that the harmful effects of smoking are reversible. This supports results of earlier studies, showing that after cessation of smoking, the negative effect on disability progression slowly decreases, independent to the cumulative dose of smoking $[8,10]$.

Our study has some limitations. Although the mean follow-up time was long (almost 5 years), there was a wide range. To overcome this, we used a COX regression model to correct for follow-up time. We also performed a sub-analysis, where CIS patients with less than 2 years of follow-up were excluded. Excluding these patients left 200 patients for analysis and did not change our results. Yet, for demonstration of an association between smoking at time of CIS and later disability (EDSS) a longer follow-up would be needed.

Second, there is a possibility that our results are explained by potential confounding lifestyle factors such as body mass index (BMI) or alcohol use. It has been shown that obesity is a risk factor for MS [29]. However, it is not likely that a high BMI explained the effect seen here, as obesity is more common in non-smokers [30]. A Swedish study showed an inverse association of alcohol consumption with MS [31]. We did not observe an effect of alcohol use on CDMS diagnosis in the regression analysis (data not shown).

Third, a follow-up MRI scan was not performed according to a fixed protocol. Instead, we used the classic Poser criteria that are based on clinical manifestations to define CDMS. Thus, we can only claim an association with clinical disease activity but not with lesion accrual on MRI scan.

It is not likely that CIS patients with a second attack during follow-up had over-reported smoking at time of CIS. Even in case recall would play a serious role here, recall of smoking would be expectedly more strong for the question of past smoking [32]. Yet, it was only recent smoking, more plausibly related to concurrent biological processes just before, during and after the first demyelinating attack, that showed an association.

The exact influence of ongoing smoking on the progression of the auto-inflammatory process around a first clinical attack of demyelination remains to be determined. It may involve several pathways, including both direct and indirect influences of tobacco toxins and smoke particles on $\mathrm{T}$ cells and antigen presenting cells [33].

To conclude, we show in a large prospective cohort of CIS patients that smoking at time of CIS is an independent risk factor for a future CDMS diagnosis. Smoking status could even be a relevant parameter in predictive models on a possible MS disease course after CIS. Since smoking is a modifiable risk factor, our study draws attention to the relevance of counselling patients about smoking. Though intervention studies will be difficult to execute, this study may provide evidence for the argument to quit smoking for patients with a first attack of suspected MS.

Acknowledgements The authors thank the patients who participated in the PROUD study, and the physicians who helped with the data collection.

Funding This study was supported by the Dutch MS Research Foundation.

Conflicts of interest Roos M. van der Vuurst de Vries-declares no conflict of interests. Julia Y. Mescheriakova-declares no conflict of interests. Tessel F. Runia-declares no conflict of interests. Theodora A. M. Siepman-declares no conflict of interests. Beatrijs H. A. Wokke-declares no conflict of interests. Johnny P. A. Samijn received honoraria for serving on advisory boards for Merck-Serono, Genzyme and Roche. And received travel Grants from Merck-Serono. He participated in trials with BiogenIdec, Merck-Serono, Roche and Genzyme, outside the submitted work. Rogier Q. Hintzen received honoraria for serving on advisory boards for Biogen Idec, Roche, Sanofi. He participated in trials with BiogenIdec, Merck-Serono, Roche, Genzyme and Novartis, outside the submitted work.

Ethical standards This study was approved by the Medical Ethics Committee of Erasmus MC Rotterdam, the Netherlands.

Informed consent Written informed consent was obtained from all patients.

Open Access This article is distributed under the terms of the Creative Commons Attribution 4.0 International License (http://creativeco mmons.org/licenses/by/4.0/), which permits unrestricted use, distribution, and reproduction in any medium, provided you give appropriate credit to the original author(s) and the source, provide a link to the Creative Commons license, and indicate if changes were made.

\section{References}

1. Compston A, Coles A (2008) Multiple sclerosis. Lancet 372(9648):1502-1517

2. Huynh JL, Casaccia P (2013) Epigenetic mechanisms in multiple sclerosis: implications for pathogenesis and treatment. Lancet Neurol 12(2):195-206

3. Frischer JM, Bramow S, Dal-Bianco A et al (2009) The relation between inflammation and neurodegeneration in multiple sclerosis brains. Brain 132(Pt 5):1175-1189

4. Hedstrom AK, Hillert J, Olsson T, Alfredsson L (2013) Smoking and multiple sclerosis susceptibility. Eur $\mathrm{J}$ Epidemiol 28(11):867-874

5. Handel AE, Williamson AJ, Disanto G et al (2011) Smoking and multiple sclerosis: an updated meta-analysis. PLoS One 6(1):e16149 
6. Hedstrom AK, Baarnhielm M, Olsson T, Alfredsson L (2011) Exposure to environmental tobacco smoke is associated with increased risk for multiple sclerosis. Mult Scler 17(7):788-793

7. Hernan MA, Jick SS, Logroscino G et al (2005) Cigarette smoking and the progression of multiple sclerosis. Brain $128(\mathrm{Pt}$ 6): $1461-1465$

8. Ramanujam R, Hedstrom AK, Manouchehrinia A et al (2015) Effect of smoking cessation on multiple sclerosis prognosis. JAMA Neurol 72(10):1117-1123

9. Manouchehrinia A, Tench CR, Maxted J et al (2013) Tobacco smoking and disability progression in multiple sclerosis: United Kingdom cohort study. Brain 136(Pt 7):2298-2304

10. Tanasescu R, Constantinescu CS, Tench CR, Manouchehrinia A (2017) Smoking cessation and the reduction of disability progression in Multiple Sclerosis: a cohort study. Nicotine Tob Res. https ://doi.org/10.1093/ntr/ntx084

11. Hedstrom AK, Sundqvist E, Baarnhielm M et al (2011) Smoking and two human leukocyte antigen genes interact to increase the risk for multiple sclerosis. Brain 134(Pt 3):653-664

12. Hedstrom AK, Bomfim IL, Barcellos LF et al (2014) Interaction between passive smoking and two HLA genes with regard to multiple sclerosis risk. Int J Epidemiol 43(6):1791-1798

13. Miller DH, Chard DT, Ciccarelli O (2012) Clinically isolated syndromes. Lancet Neurol 11(2):157-169

14. Arikanoglu A, Shugaiv E, Tuzun E, Eraksoy M (2013) Impact of cigarette smoking on conversion from clinically isolated syndrome to clinically definite multiple sclerosis. Int $\mathbf{J}$ Neurosci 123(7):476-479

15. Di Pauli F, Reindl M, Ehling R et al (2008) Smoking is a risk factor for early conversion to clinically definite multiple sclerosis. Mult Scler 14(8):1026-1030

16. Correale J, Farez MF (2015) Smoking worsens multiple sclerosis prognosis: two different pathways are involved. J Neuroimmunol 281:23-34

17. Munger KL, Fitzgerald KC, Freedman MS et al (2015) No association of multiple sclerosis activity and progression with EBV or tobacco use in BENEFIT. Neurology 85(19):1694-1701

18. Horakova D, Zivadinov R, Weinstock-Guttman B et al (2013) Environmental factors associated with disease progression after the first demyelinating event: results from the multi-center SET study. PLoS One 8(1):e53996

19. Miller DH, Weinshenker BG, Filippi M et al (2008) Differential diagnosis of suspected multiple sclerosis: a consensus approach. Mult Scler 14(9):1157-1174
20. Schumacher GA, Beebe G, Kibler RF et al (1965) Problems of experimental trials of therapy in multiple sclerosis: report by the panel on the evaluation of experimental trials of therapy in multiple sclerosis. Ann N Y Acad Sci 122:552-568

21. Poser CM, Paty DW, Scheinberg L et al (1983) New diagnostic criteria for multiple sclerosis: guidelines for research protocols. Ann Neurol 13(3):227-231

22. Kurtzke JF (1983) Rating neurologic impairment in multiple sclerosis: an expanded disability status scale (EDSS). Neurology 33(11):1444-1452

23. Versini M, Tiosano S, Comaneshter D et al (2017) Smoking and obesity in systemic lupus erythematosus: a cross-sectional study. Eur J Clin Invest 47(6):422-427

24. Stolt P, Bengtsson C, Nordmark B et al (2003) Quantification of the influence of cigarette smoking on rheumatoid arthritis: results from a population based case-control study, using incident cases. Ann Rheum Dis 62(9):835-841

25. Degelman ML, Herman KM (2017) Smoking and multiple sclerosis: a systematic review and meta-analysis using the Bradford Hill criteria for causation. Mult Scler Relat Disord 17:207-216

26. Kappos L, Polman CH, Freedman MS et al (2006) Treatment with interferon beta- $1 \mathrm{~b}$ delays conversion to clinically definite and McDonald MS in patients with clinically isolated syndromes. Neurology 67(7):1242-1249

27. Tintore M, Rovira A, Rio J et al (2015) Defining high, medium and low impact prognostic factors for developing multiple sclerosis. Brain 138(Pt 7):1863-1874

28. Sayao AL, Devonshire V, Tremlett H (2007) Longitudinal follow-up of "benign" multiple sclerosis at 20 years. Neurology 68(7):496-500

29. Munger KL, Chitnis T, Ascherio A (2009) Body size and risk of MS in two cohorts of US women. Neurology 73(19):1543-1550

30. Dare S, Mackay DF, Pell JP (2015) Relationship between smoking and obesity: a cross-sectional study of 499,504 middle-aged adults in the UK general population. PLoS One 10(4):e0123579

31. Hedstrom AK, Hillert J, Olsson T, Alfredsson L (2014) Alcohol as a modifiable lifestyle factor affecting multiple sclerosis risk. JAMA Neurol 71(3):300-305

32. Shiffman S, Hufford M, Hickcox M et al (1997) Remember that? A comparison of real-time versus retrospective recall of smoking lapses. J Consult Clin Psychol 65(2):292-300

33. Arnson Y, Shoenfeld Y, Amital H (2010) Effects of tobacco smoke on immunity, inflammation and autoimmunity. J Autoimmun 34(3):J258-J265 